NISSUNA UMANA INVESTIGAZIONE SI PUO DIMANDARE VERA SCIENZIA S'ESSA NON PASSA PER LE MATEMATICHE DIMOSTRAZIONI LEONARDO DA VINCI

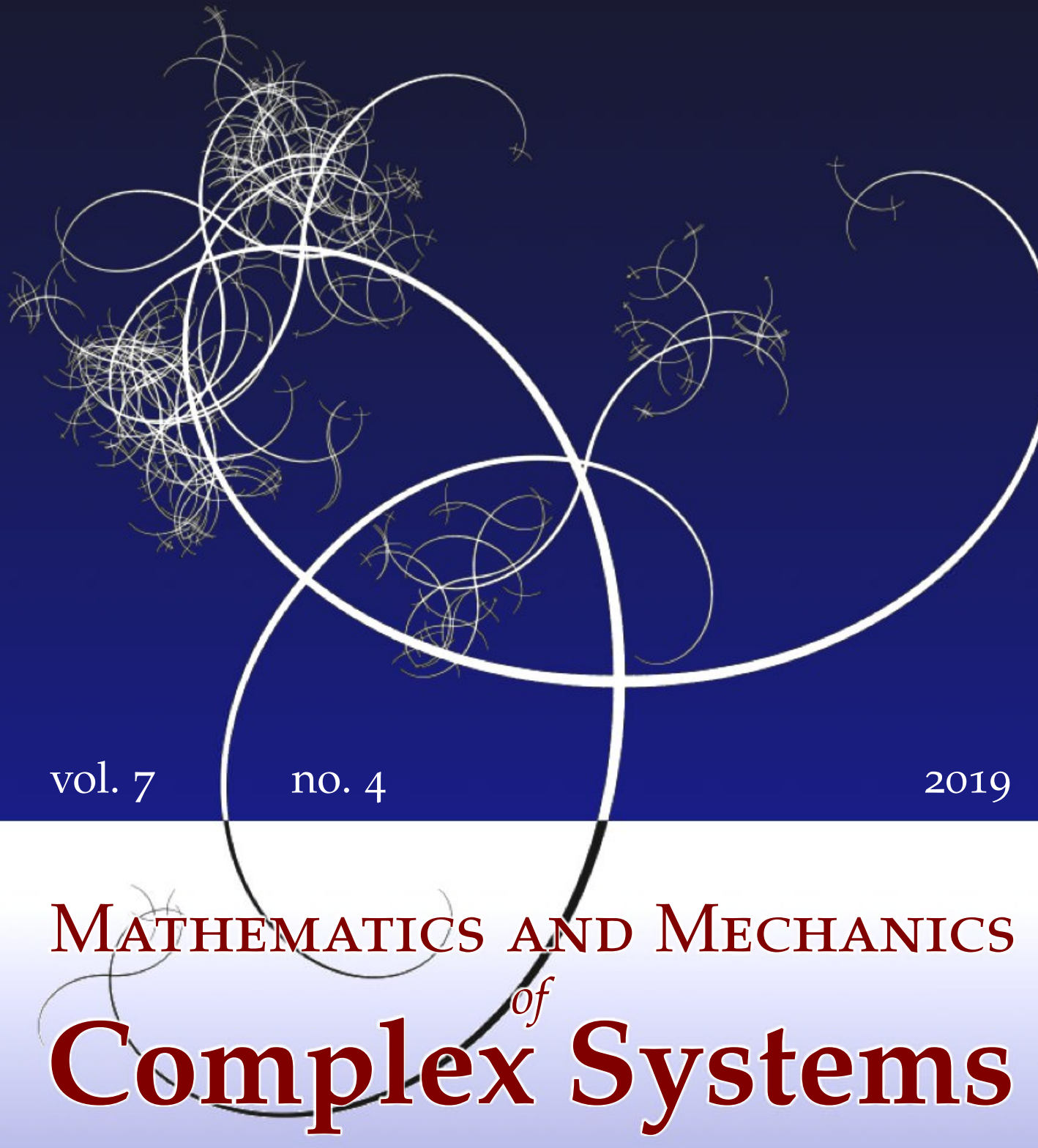

IVAN GIORGIO, NiCOLA L. RiZZI,

Ugo Andreaus and David J. Steigmann

A TWO-DIMENSIONAL CONTINUUM MODEL OF PANTOGRAPHIC SHEETS MOVING IN A 3D SPACE

AND ACCOUNTING FOR THE OFFSET AND RELATIVE ROTATIONS OF THE FIBERS 


\title{
A TWO-DIMENSIONAL CONTINUUM MODEL OF PANTOGRAPHIC SHEETS MOVING IN A 3D SPACE AND ACCOUNTING FOR THE OFFSET AND RELATIVE ROTATIONS OF THE FIBERS
}

\author{
IVAN Giorgio, Nicola L. RizZI, \\ Ugo Andreaus and David J. SteigmanN
}

\begin{abstract}
Recently growing attention has been paid to the particular class of metamaterials which has been called pantographic. Pantographic metamaterials have the following peculiar features: (i) their continuum model, at the macroscale, has to include a term of the deformation energy depending on the second gradient of placement, (ii) they can show an elastic behavior in large deformation regimes, and (iii) they are resilient and tough during rupture phenomena (dell' Isola et al. 2015). In order to predict pantographic metamaterials' mechanical behavior, it is possible to introduce a three-dimensional continuum micromodel, in which their internal geometrical microstructure is described in detail. However, the computational costs of this choice are presently prohibitive. In this paper, we introduce a reduced order model for pantographic sheets - which are an example of an elastic surface - whose kinematics include, for each of the two constituting families of fibers fully independent three-dimensional (i) placement and (ii) rotation fields. In this way it is possible to include, also in the reduced order model, (i) the initial and the actual offset between the fibers and (ii) the deformation energy of the interconnecting pivots. By postulating a simplified expression for the deformation energy we prove that also a reduced order model can describe some experimental observed buckling and postbuckling phenomena. The promising results which we present here motivate the quest of more general expressions for deformation energy capable of capturing the fully nonlinear behavior exhibited by pantographic sheets.
\end{abstract}

\section{Introduction}

Metamaterials are materials with microstructures that have been designed to achieve novel macroscopic behavior tailored to some specific uses or having some specific

\section{Communicated by Victor A. Eremeyev.}

Giorgio is the corresponding author.

MSC2010: 74KXX, 74QXX.

Keywords: elastic surface theory, second-gradient models, pantographic structures. 


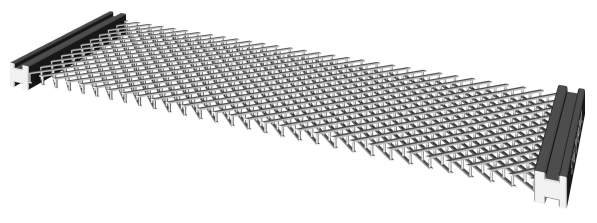

Figure 1. A sample of pantographic material.

features [Barchiesi et al. 2019b; dell'Isola et al. 2019]. In particular, pantographic metamaterials [Alibert et al. 2003; Seppecher et al. 2011; Eugster et al. 2019] possess some very peculiar characteristics. At the macroscale they entail deformation energies that typically depend only on the second gradient of placement. Pantographic metamaterials having deformation energies depending "mainly" on the second gradient of placement [Alibert and Della Corte 2019] are capable of large deformations that remain in an elastic regime and exhibit great toughness in rupture phenomena [Placidi et al. 2016; Turco et al. 2016b; De Angelo et al. $2019 \mathrm{~b}$ ]. Of course, to predict the behavior of pantographic metamaterials, it is possible to introduce a micromodel, where their internal microstructured architecture is fully described, in all its geometrical and mechanical complexity, by a conventional three-dimensional continuum. It is clear, on the other hand, that the computational burden implied by this modeling choice can be prohibitive, the availability of modern computing tools notwithstanding. Indeed, despite having optimized codes of this kind [Giorgio 2016], it is not feasible to find the solutions of structural optimization problems by employing, as subroutines, the codes calculating the equilibrium shapes of aforementioned three-dimensional continua. Therefore, the question arises: can we find a reduced order model with sufficient resolution and which is computationally convenient?

A first simplification can be achieved by limiting our attention to two-dimensional pantographic metamaterials, called also pantographic sheets. This has been considered in a series of papers [dell'Isola et al. 2015; 2019; Eremeyev et al. 2018; Barchiesi and Placidi 2017; Steigmann and dell'Isola 2015; Andreaus et al. 2018] where a specific class of two-dimensional continua, generalizing standard KirchhoffLove plate theory, is considered. In these models, the deformation energy depends not only on the second gradient of the transverse component of displacement, as in standard plate theory, but also on the second gradient of in-plane displacement.

Nevertheless, despite these advances, such models do not take into account some important mechanical features of pantographic sheets.

In particular, as shown in [De Angelo et al. 2019a], the mechanical behavior of pantographic sheets is affected by the deformation of the interconnecting pivots (see Figure 1) and on the (deformation-dependent) offset between the constituent fibers [Barchiesi et al. 2019a]. This phenomenology has yet to be modeled explicitly, 

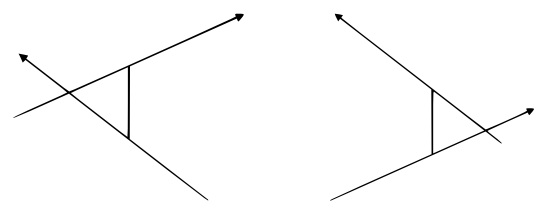

Figure 2. Schematic representation of a connection: "lefthanded" and "right-handed", respectively.

except in the case of purely planar deformation [Spagnuolo et al. 2017]. On the other hand, it has been estimated, by using complex numerical simulations based on three-dimensional continuum modeling, that there is a large amount of deformation energy which is stored in pivots and nearby material points [Giorgio 2016]. Moreover, in some deformation processes, the offset between the fibers, together with their relative rotations, play a significant role in buckling and postbuckling phenomena. This suggests a form of chirality of pantographic sheets, i.e., some sheets are "left-handed" while others are "right-handed", due to the offset of constituent fibers (see Figure 2) and to the relative orientation of upper and lower arrays of fibers.

The aim of this paper is to present a reduced order model for pantographic sheets that accounts for the interplay among chirality, offset between the constituent fibers, and the deformation of interconnecting pivots play.

The reduced order continuum model for pantographic sheets discussed in this work has enriched kinematics, including:

(1) two distinct placement fields, one for each fiber family (the upper and the lower) constituting the sheet,

(2) two distinct rotation fields, describing the rotations of the sections of each family of fibers.

The enriched kinematics allows us to include, in the proposed "richer" reduced order model:

(1) the initial and the actual offset between the upper and lower families of fibers,

(2) the deformation energy stored in the interconnecting pivots.

The importance of these two mechanical properties of pantographic sheets has been emphasized in [Giorgio 2016] where it is proved, using simulations based on a detailed 3D first-gradient microcontinuum model, that in many deformation processes the separation distance of the fibers varies significantly and that a large percentage of deformation energy is stored in the small volumes containing the interconnecting pivots.

The reduced order model is then completed by postulating an expression for the deformation energy in terms of those deformation measures which are naturally introduced in terms of the proposed kinematical descriptors. 
Further, we postulate a simple expression for the deformation energy of the pivots which is physically well motivated when pivots undergo small deformations. By using this simplified deformation energy we show that the reduced order model can describe, at least in a qualitative way, the buckling and postbuckling phenomena that have been observed experimentally and predicted numerically.

To this end we compare the predictions of the present model with numerical simulations based on a refined three-dimensional continuum model, obtained with codes and numerical procedures which are further optimized relative to those used in [Giorgio 2016; De Angelo et al. 2019a; Cazzani et al. 2018a; 2018b].

The quantitative agreement is lost when interconnecting pivots undergo large deformations. This circumstance leads us to postulate more complex expressions for the deformation energy. Indeed our results suggest the structure and the properties of more general constitutive relations for the deformation energy aimed at describing the fully nonlinear behavior exhibited by pantographic sheets.

\section{Fiber kinematics}

Figure 3 depict the fiber directions in the pantographic sheet together with the local bases. In what follows all the components will be given in the global base $\boldsymbol{b}_{i}$. To describe fibers kinematics we introduce the fields

$$
\begin{array}{ll}
\text { fiber }_{1}: & { }^{1} \boldsymbol{u},{ }^{1} \boldsymbol{R}, \\
\text { fiber }_{2}: & { }^{2} \boldsymbol{u},{ }^{2} \boldsymbol{R},
\end{array}
$$

where the vector fields ${ }^{1} \boldsymbol{u}$ and ${ }^{2} \boldsymbol{u}$ are the displacements of the beam axes, and ${ }^{1} \boldsymbol{R}$ and ${ }^{2} \boldsymbol{R}$ are orthogonal tensor fields that represent the rotations of the beam crosssections (for a more comprehensive discussion, see [Eremeyev and Pietraszkiewicz 2016; 2006; Eremeyev 2018; 2019]). The components of these fields are

$$
\begin{array}{cc}
{ }^{1} \boldsymbol{u}={ }^{1} u_{i} \boldsymbol{b}_{i}, & { }^{2} \boldsymbol{u}={ }^{2} u_{i} \boldsymbol{b}_{i}, \\
{ }^{1} \boldsymbol{R}={ }^{1} R_{i j} \boldsymbol{b}_{i} \otimes \boldsymbol{b}_{j}, \quad{ }^{2} \boldsymbol{R}={ }^{2} \widetilde{R}_{i j} \boldsymbol{c}_{i} \otimes \boldsymbol{c}_{j}={ }^{2} R_{i j} \boldsymbol{b}_{i} \otimes \boldsymbol{b}_{j} .
\end{array}
$$

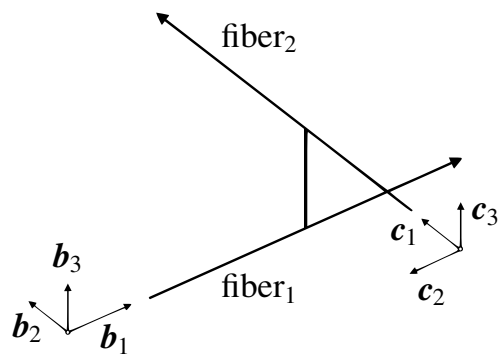

Figure 3. Reference frames. 
The components ${ }^{1} R_{i j}$ are given in terms of the Bryant angles ${ }^{1} \varphi_{1},{ }^{1} \varphi_{2}$, and ${ }^{1} \varphi_{3}$ and ${ }^{2} \widetilde{R}_{i j}$ are given in terms of ${ }^{2} \varphi_{1},{ }^{2} \varphi_{2}$, and ${ }^{2} \varphi_{3}$.

The deformation measures are

$$
\begin{aligned}
{ }^{\alpha} \boldsymbol{e} & ={ }^{\alpha} \boldsymbol{R}^{\top}\left({ }^{\alpha} \boldsymbol{X}^{\prime}+{ }^{\alpha} \boldsymbol{u}^{\prime}\right)-{ }^{\alpha} \boldsymbol{X}^{\prime}, \\
{ }^{\alpha} \boldsymbol{\kappa} & =\left({ }^{\alpha} \boldsymbol{R}^{\top \alpha} \boldsymbol{R}^{\prime}\right)_{\times},
\end{aligned}
$$

where $\alpha=\{1,2\},{ }^{\alpha} \boldsymbol{X}$ are the position of the beam axes in the reference configuration, the prime is used to denote differentiation with respect to the abscissa along the axes, and ${ }^{\alpha} \boldsymbol{\kappa}$ is the axial vector of the skew tensor ${ }^{\alpha} \boldsymbol{R}^{\top \alpha}{ }^{\prime} \boldsymbol{R}^{\prime}$.

The components of ${ }^{\alpha} \boldsymbol{e}$ and ${ }^{\alpha} \boldsymbol{\kappa}$ in the global basis are

$$
\begin{aligned}
& { }^{1} \varepsilon={ }^{1} e_{1}=-1+\left(1+{ }^{1} u_{1,1}\right) \cos { }^{1} \varphi_{2} \cos { }^{1} \varphi_{3} \\
& +{ }^{1} u_{2,1}\left(\cos { }^{1} \varphi_{3} \sin ^{1} \varphi_{1} \sin ^{1} \varphi_{2}+\cos { }^{1} \varphi_{1} \sin ^{1} \varphi_{3}\right) \\
& +{ }^{1} u_{3,1}\left(\sin ^{1} \varphi_{1} \sin ^{1} \varphi_{3}-\cos ^{1} \varphi_{1} \cos { }^{1} \varphi_{3} \sin ^{1} \varphi_{2}\right), \\
& { }^{1} \gamma_{p}={ }^{1} e_{2}=-\left(1+{ }^{1} u_{1,1}\right) \cos { }^{1} \varphi_{2} \sin ^{1} \varphi_{3} \\
& +{ }^{1} u_{2,1}\left(\cos { }^{1} \varphi_{1} \cos { }^{1} \varphi_{3}-\sin ^{1} \varphi_{1} \sin ^{1} \varphi_{2} \sin ^{1} \varphi_{3}\right) \\
& +{ }^{1} u_{3,1}\left(\cos { }^{1} \varphi_{3} \sin ^{1} \varphi_{1}+\cos ^{1} \varphi_{1} \sin ^{1} \varphi_{2} \sin ^{1} \varphi_{3}\right) \text {, } \\
& { }^{1} \gamma_{n}={ }^{1} e_{3}=\left(1+{ }^{1} u_{1,1}\right) \sin { }^{1} \varphi_{2}-{ }^{1} u_{2,1} \cos { }^{1} \varphi_{2} \sin { }^{1} \varphi_{1}+{ }^{1} u_{3,1} \cos { }^{1} \varphi_{1} \cos { }^{1} \varphi_{2} \text {, } \\
& { }^{1} \kappa_{1}={ }^{1} \kappa_{\tau}={ }^{1} \varphi_{1,1} \cos { }^{1} \varphi_{2} \cos { }^{1} \varphi_{3}+{ }^{1} \varphi_{2,1} \sin { }^{1} \varphi_{3} \text {, } \\
& { }^{1} \kappa_{2}={ }^{1} \kappa_{n}={ }^{1} \varphi_{1,1} \cos { }^{1} \varphi_{2} \sin ^{1} \varphi_{3}-{ }^{1} \varphi_{2,1} \cos { }^{1} \varphi_{3} \text {, } \\
& { }^{1} \kappa_{3}={ }^{1} \kappa_{g}={ }^{1} \varphi_{1,1} \sin ^{1} \varphi_{2}+{ }^{1} \varphi_{3,1} \text {, } \\
& { }^{2} \varepsilon={ }^{2} e_{2}=-1-{ }^{2} u_{1,2}\left(\cos ^{2} \varphi_{3} \sin ^{2} \varphi_{1} \sin ^{2} \varphi_{2}+\cos ^{2} \varphi_{1} \sin ^{2} \varphi_{3}\right) \\
& +\left(1+{ }^{2} u_{2,2}\right) \cos ^{2} \varphi_{2} \cos ^{2} \varphi_{3} \\
& +{ }^{2} u_{3,2}\left(\sin ^{2} \varphi_{1} \sin ^{2} \varphi_{3}-\cos ^{2} \varphi_{1} \cos ^{2} \varphi_{3} \sin ^{2} \varphi_{2}\right), \\
& { }^{2} \gamma_{p}={ }^{2} e_{1}={ }^{2} u_{1,2}\left(\cos ^{2} \varphi_{1} \cos ^{2} \varphi_{3}-\sin ^{2} \varphi_{1} \sin ^{2} \varphi_{2} \sin ^{2} \varphi_{3}\right) \\
& +\left(1+{ }^{2} u_{2,2}\right) \cos ^{2} \varphi_{2} \sin ^{2} \varphi_{3} \\
& -{ }^{2} u_{3,2}\left(\cos ^{2} \varphi_{3} \sin ^{2} \varphi_{1}+\cos ^{2} \varphi_{1} \sin ^{2} \varphi_{2} \sin ^{2} \varphi_{3}\right) \text {, } \\
& { }^{2} \gamma_{n}={ }^{2} e_{3}={ }^{2} u_{1,2} \cos ^{2} \varphi_{2} \sin ^{2} \varphi_{1}+\left(1+{ }^{2} u_{2,2}\right) \sin ^{2} \varphi_{2}+{ }^{2} u_{3,2} \cos { }^{2} \varphi_{1} \cos ^{2} \varphi_{2} \text {, } \\
& { }^{2} \kappa_{1}={ }^{2} \kappa_{n}=-{ }^{2} \varphi_{1,2} \cos { }^{2} \varphi_{2} \sin ^{2} \varphi_{3}+{ }^{2} \varphi_{2,2} \cos ^{2} \varphi_{3} \text {, } \\
& { }^{2} \kappa_{2}={ }^{2} \kappa_{\tau}={ }^{2} \varphi_{1,2} \cos { }^{2} \varphi_{2} \cos { }^{2} \varphi_{3}+{ }^{2} \varphi_{2,2} \sin ^{2} \varphi_{3} \text {, } \\
& { }^{2} \kappa_{3}={ }^{2} \kappa_{g}={ }^{2} \varphi_{1,2} \sin ^{2} \varphi_{2}+{ }^{2} \varphi_{3,2} \text {, }
\end{aligned}
$$

where $(\cdot)_{, 1}=\frac{\partial}{\partial s_{1}}$ and $(\cdot)_{, 2}=\frac{\partial}{\partial s_{2}}$. 


\section{Kinematics of the pivots}

A typical pivot has been assumed to be a cylinder of radius $r$ and height $h$. The two bases of the pivot are assumed to be rigid bodies whose motion is described by a vector and an orthogonal tensor. The vector describes the displacement of a selected point (say $\boldsymbol{X}$ ) of the body and the tensor the rotation of the base (see Figure 4).

Henceforth, quantities related to each one of the two bases will be denoted by the indices $1 p$ and $2 p$, respectively.

Now, if

$$
{ }^{1 p} \boldsymbol{X}, \quad{ }^{2 p} \boldsymbol{X}
$$

are the selected points on the bases, we will denote by

$$
{ }^{1 p} \boldsymbol{u}\left({ }^{1 p} \boldsymbol{X}\right), \quad{ }^{2 p} \boldsymbol{u}\left({ }^{2 p} \boldsymbol{X}\right)
$$

the displacements of those points and by

$$
{ }^{1 p} \boldsymbol{R}, \quad{ }^{2 p} \boldsymbol{R}
$$

the rotations of the bases.

The deformation measures of the pivot are assumed as

$$
\begin{aligned}
{ }^{p} \boldsymbol{e} & ={ }^{1 p} \boldsymbol{R}^{\top}\left({ }^{2 p} \boldsymbol{u}-{ }^{1 p} \boldsymbol{u}\right)+\left({ }^{1 p} \boldsymbol{R}^{\top}-\boldsymbol{I}\right)\left({ }^{2 p} \boldsymbol{X}-{ }^{1 p} \boldsymbol{X}\right), \\
{ }^{p} \boldsymbol{E} & =\operatorname{skw}\left({ }^{1 p} \boldsymbol{R}^{\top}{ }^{2 p} \boldsymbol{R}\right)
\end{aligned}
$$

and can be proved to be frame-invariant or objective. The deformation measure related to ${ }^{p} \boldsymbol{E}$ is represented by its axial vector:

$$
{ }^{p} \boldsymbol{\kappa}={ }^{p} \boldsymbol{E}_{\times} .
$$

By considering the orthogonal tensor

$$
\boldsymbol{Q}=Q_{\alpha}{ }_{\beta} \boldsymbol{b}_{\alpha} \otimes \boldsymbol{b}_{\beta}
$$

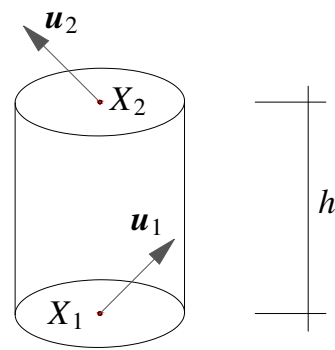

Figure 4. Pivot. 

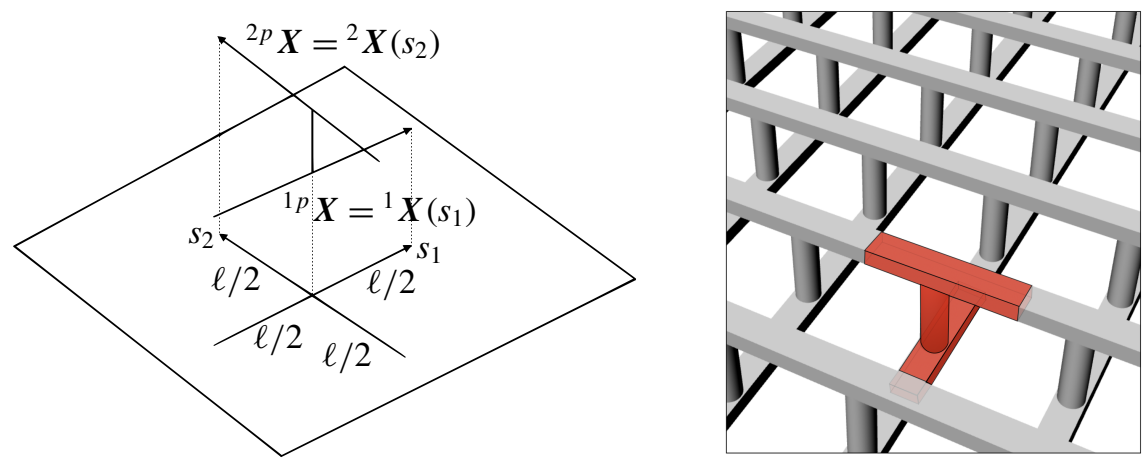

Figure 5. Unit cell.

which defines the basis transformation

$$
\boldsymbol{c}_{\alpha}=Q_{\beta \alpha} \boldsymbol{b}_{\beta}
$$

that in our case is

$$
\left(\begin{array}{l}
\boldsymbol{c}_{1} \\
\boldsymbol{c}_{2} \\
\boldsymbol{c}_{3}
\end{array}\right)=\left(\begin{array}{lll}
\boldsymbol{b}_{1} & \boldsymbol{b}_{2} & \boldsymbol{b}_{3}
\end{array}\right)\left(\begin{array}{rrr}
0 & -1 & 0 \\
1 & 0 & 0 \\
0 & 0 & 1
\end{array}\right),
$$

we obtain

$$
\begin{aligned}
{ }^{p} \boldsymbol{e} & =\left[{ }^{1 p} R_{j i}\left({ }^{2 p} u_{j}-{ }^{1 p} u_{j}\right)+\left({ }^{1 p} R_{j i}-\delta_{i j}\right)\left({ }^{2 p} X_{j}-{ }^{1 p} X_{j}\right)\right] \boldsymbol{b}_{i}, \\
{ }^{p} \boldsymbol{E} & =\operatorname{skw}\left({ }^{1 p} R_{\alpha i} Q_{\alpha \gamma}{ }^{2 p} \widetilde{R}_{\gamma \beta} Q_{j \beta} \boldsymbol{b}_{i} \otimes \boldsymbol{b}_{j}\right), \\
{ }^{p} \boldsymbol{\kappa} & ={ }^{p} E_{32} \boldsymbol{b}_{1}+{ }^{p} E_{13} \boldsymbol{b}_{2}+{ }^{p} E_{21} \boldsymbol{b}_{3} .
\end{aligned}
$$

\section{2D continuum kinematics}

The elementary cell is shown in Figure 5, where $\ell$ is the space between the two neighboring pivots. We make the identifications

$$
\begin{aligned}
{ }^{1 p} \boldsymbol{X} & ={ }^{1} \boldsymbol{X}\left(s_{1}\right), & { }^{2 p} \boldsymbol{X} & ={ }^{2} \boldsymbol{X}\left(s_{2}\right), \\
{ }^{1 p} \boldsymbol{u}\left({ }^{1 p} \boldsymbol{X}\right) & ={ }^{1} \boldsymbol{u}\left(s_{1}\right), & { }^{2 p} \boldsymbol{u}\left({ }^{2 p} \boldsymbol{X}\right) & ={ }^{2} \boldsymbol{u}\left(s_{2}\right), \\
{ }^{1 p} \boldsymbol{R} & ={ }^{1} \boldsymbol{R}\left(s_{1}\right), & { }^{2 p} \boldsymbol{R} & ={ }^{2} \boldsymbol{R}\left(s_{2}\right) .
\end{aligned}
$$

\section{2D strain energy}

The strain energy of a unit cell can be expressed as the sum of two contributions, specifically: 
(1) the term related to the fibers

$$
\begin{array}{r}
{ }^{f} \Pi_{c}=\frac{1}{2} \sum_{\alpha=1,2} \int_{-\ell / 2}^{\ell / 2}\left(\mathrm{E}^{f} A^{\alpha} \varepsilon^{2}+\mathrm{k}_{p} G^{f_{A}{ }^{\alpha} \gamma_{p}^{2}+\mathrm{k}_{n} G^{f} A^{\alpha} \gamma_{n}^{2}}\right. \\
\left.+\mathrm{q} G^{f} J_{\tau}{ }^{\alpha} \kappa_{\tau}^{2}+\mathrm{E} J_{n}{ }^{\alpha} \kappa_{n}^{2}+\mathrm{E} J_{g}{ }^{\alpha} \kappa_{g}^{2}\right) \mathrm{d} s_{\alpha}
\end{array}
$$

where $\mathrm{E}$ and $G$ are the Young and shear moduli, respectively, ${ }^{f} A$ is the crosssection area, $J_{g}$ is the moment of geodesic flexural inertia, $J_{n}$ is the moment of normal flexural inertia, ${ }^{f} J_{\tau}$ is the polar moment inertia, and $\mathrm{k}_{p}, \mathrm{k}_{n}$, and $q$ are positive constants,

(2) the term related to the pivot connecting the fibers

${ }^{p} \Pi_{c}=\frac{1}{2 h}\left(\mathrm{E}^{p} A^{p} e_{3}{ }^{2}+4 \mathrm{E}^{p} J\left({ }^{p} \kappa_{1}{ }^{2}+{ }^{p} \kappa_{2}{ }^{2}\right)+G^{p} J_{\tau}{ }^{p} \kappa_{3}{ }^{2}+\frac{12 \mathrm{E}^{p} J}{h^{2}}\left({ }^{p} e_{1}{ }^{2}+{ }^{p} e_{2}{ }^{2}\right)\right)$

where ${ }^{p} A,{ }^{p} J_{\tau}$, and ${ }^{p} J$ are the area and the torsional and flexural inertial moments of the cross-area of the pivot, respectively.

The density of the strain energy of the two-dimensional elastic surface is defined as the average strain energy per unit cell, i.e.,

$$
\pi=\pi_{f}+\pi_{p}=\frac{1}{{ }^{c} A}\left({ }^{f} \Pi_{c}+{ }^{p} \Pi_{c}\right)
$$

where ${ }^{c} A$ is the two-dimensional measure of the unit cell, i.e., $\ell^{2}$. This makes explicit the contributions of the fibers $\pi_{f}$ and of the pivots $\pi_{p}$. Having in mind an asymptotic homogenization procedure, we can imagine an infinite distribution of fibers on the area $\ell^{2}$ along both the original directions of the discrete net of fibers and express the homogenized strain energy as

$$
\begin{aligned}
\pi_{f}=\frac{1}{\ell^{2}} \sum_{\alpha=1,2} \frac{\ell}{2}\left(\mathrm{E}^{f} A^{\alpha} \varepsilon^{2}+\mathrm{k}_{p} G^{f} A^{\alpha} \gamma_{p}^{2}+\mathrm{k}_{n} G^{f} A^{\alpha} \gamma_{n}^{2}\right. \\
\left.+\mathrm{q} G^{f} J_{\tau}{ }^{\alpha} \kappa_{\tau}^{2}+\mathrm{E} J_{n}{ }^{\alpha} \kappa_{n}^{2}+\mathrm{E} J_{g}{ }^{\alpha} \kappa_{g}^{2}\right)
\end{aligned}
$$

and

$$
\pi_{p}=\frac{1}{2 \ell^{2} h}\left(\mathrm{E}^{p} A^{p} e_{3}{ }^{2}+4 \mathrm{E}^{p} J\left({ }^{p} \kappa_{1}{ }^{2}+{ }^{p} \kappa_{2}{ }^{2}\right)+G^{p} J_{\tau}{ }^{p} \kappa_{3}{ }^{2}+\frac{12 \mathrm{E}^{p} J}{h^{2}}\left({ }^{p} e_{1}{ }^{2}+{ }^{p} e_{2}{ }^{2}\right)\right) .
$$

As a result, we have for the strain energy density

$$
\begin{gathered}
\pi_{f}=\frac{1}{2}\left[{ }^{f} \mathscr{K}_{e}\left({ }^{1} \varepsilon^{2}+{ }^{2} \varepsilon^{2}\right)+\mathscr{K}_{\gamma_{p}}\left({ }^{1} \gamma_{p}{ }^{2}+{ }^{2} \gamma_{p}^{2}\right)+\mathscr{K}_{\gamma_{n}}\left({ }^{1} \gamma_{n}{ }^{2}+{ }^{2} \gamma_{n}{ }^{2}\right)\right. \\
\left.+{ }^{f} \mathscr{K}_{\tau}\left({ }^{1} \kappa_{\tau}{ }^{2}+{ }^{2} \kappa_{\tau}{ }^{2}\right)+\mathscr{K}_{n}\left({ }^{1} \kappa_{n}{ }^{2}+{ }^{2} \kappa_{n}{ }^{2}\right)+\mathscr{K}_{g}\left({ }^{1} \kappa_{g}{ }^{2}+{ }^{2} \kappa_{g}{ }^{2}\right)\right], \\
\pi_{p}=\frac{1}{2}\left[\mathscr{K}_{b}\left({ }^{p} \kappa_{1}{ }^{2}+{ }^{p} \kappa_{2}{ }^{2}\right)+{ }^{p} \mathscr{K}_{\tau}{ }^{p} \kappa_{3}{ }^{2}+\mathscr{K}_{\gamma}\left({ }^{p} e_{1}{ }^{2}+{ }^{p} e_{2}{ }^{2}\right)+{ }^{p} \mathscr{K}_{e}{ }^{p} e_{3}{ }^{2}\right]
\end{gathered}
$$


where ${ }^{f} \mathscr{K}_{e}, \mathscr{K}_{\gamma_{p}}, \mathscr{K}_{\gamma_{n}},{ }^{f} \mathscr{K}_{\tau}, \mathscr{K}_{n}$, and $\mathscr{K}_{g}$ are positive constitutive parameters, assumed equal for the two continuous distributions of embedded beams, and representing the stiffnesses related to the elongation, shear, twisting, normal, and geodesic bending, respectively, while ${ }^{p_{\mathscr{K}}}{ }_{{ }}{ }_{\mathscr{K}_{\tau}}, \mathscr{K}_{\gamma}$, and $\mathscr{K}_{b}$ are positive constitutive parameters related to the elongation, torsion, shear, and bending stiffnesses of the pivots. In particular, we have

$$
\begin{aligned}
f_{\mathscr{K}_{e}} & =\frac{\mathrm{E}^{f} A}{\ell}, \mathscr{K}_{\gamma_{p}}=\frac{\mathrm{k}_{p} G^{f} A}{\ell}, \mathscr{K}_{\gamma_{n}}=\frac{\mathrm{k}_{n} G^{f} A}{\ell},{ }^{f} \mathscr{K}_{\tau}=\frac{\mathrm{q} G^{f} J_{\tau}}{\ell}, \mathscr{K}_{n}=\frac{\mathrm{E} J_{n}}{\ell}, \\
\mathscr{K}_{g} & =\frac{\mathrm{E} J_{g}}{\ell}, \quad p_{\mathscr{K}_{e}}=\frac{\mathrm{E}^{p} A}{\ell^{2} h}, \quad p_{\mathscr{K}_{\tau}}=\frac{G^{p} J_{\tau}}{\ell^{2} h}, \quad \mathscr{K}_{\gamma}=\frac{12 \mathrm{E}^{p} J}{\ell^{2} h^{3}}, \mathscr{K}_{b}=\frac{4 \mathrm{E}^{p} J}{\ell^{2} h} .
\end{aligned}
$$

\section{Numerical simulations and discussion}

We test the proposed model numerically to assess its predictive potential. To this end we use the principle of virtual work in the form

$$
-\delta \int_{\mathscr{B}}\left(\pi_{f}+\pi_{p}\right) \mathrm{d} \Omega+\delta \mathscr{W}^{\text {ext }}=0
$$

where $\delta \mathscr{W}^{\text {ext }}$ is the virtual external work done by the external actions acting on the displacements ${ }^{\alpha} u_{i}$ and the angles ${ }^{\alpha} \varphi_{i}$, and the integration is performed on the rectangular two-dimensional region $\mathscr{B}$. In particular, we compare the model (30) with a refined three-dimensional simulation based on a Cauchy-continuum model with a highly detailed description of the geometry of fibers and connections and a previous version of a reduced model presented in [Giorgio et al. 2017]. The size of the sample is $7 \times 21 \mathrm{~cm}$, and the fibers have a rectangular cross-section of height $1 \mathrm{~mm}$ and width $1.6 \mathrm{~mm}$. The fibers constrained at the short side are 10 in number for each direction, and therefore, their pitch is $4.95 \mathrm{~mm}$. The connections are cylinders with diameter $0.9 \mathrm{~mm}$. The considered material is a polyamide characterized by Young's modulus $1600 \mathrm{MPa}$ and Poisson's ratio 0.4. To compare these models, we numerically perform a bias test for three kinds of pantographic sheet, changing the height of the cylindrical connections. Specifically, we compare the results for pivots whose heights are $1 \mathrm{~mm}, 2.5 \mathrm{~mm}$, and $5 \mathrm{~mm}$. Of course, the deformability of the connection is largely affected by the height of the pivots, and hence, the hypotheses made in the present paper are expected to be more appropriate as the height of these connections increases. Figure 6 shows the plots of the longitudinal reactions obtained in the bias extension test by imposing a displacement in the direction of the length of the sample for different heights of the connections and for the three models considered: (1) the detailed Cauchy model, (2) the latent internal microstructure two-dimensional model [Giorgio et al. 2017] in which the sole kinematical mode of pivot deformation is torsion, and (3) the present twodimensional model with enhanced deformation capabilities. As expected, the new 


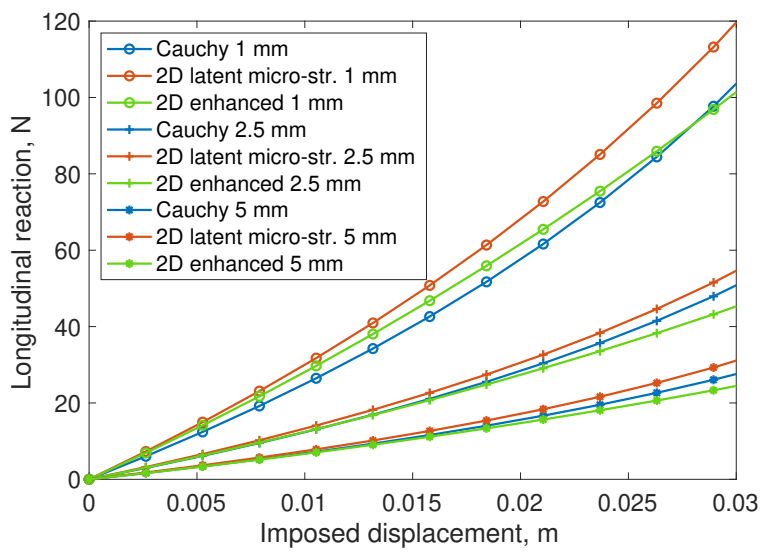

Figure 6. Reaction versus displacements using different models.
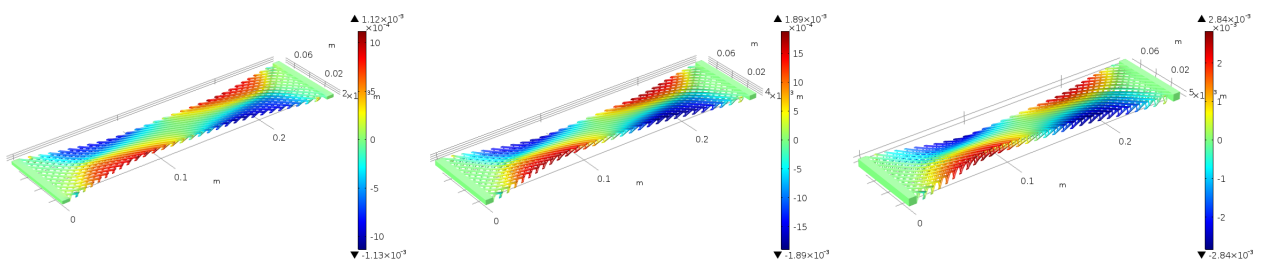

Figure 7. Cauchy 3D model with pivot heights $1 \mathrm{~mm}, 2.5 \mathrm{~mm}$, and $5 \mathrm{~mm}$. The colors show the transversal displacement.
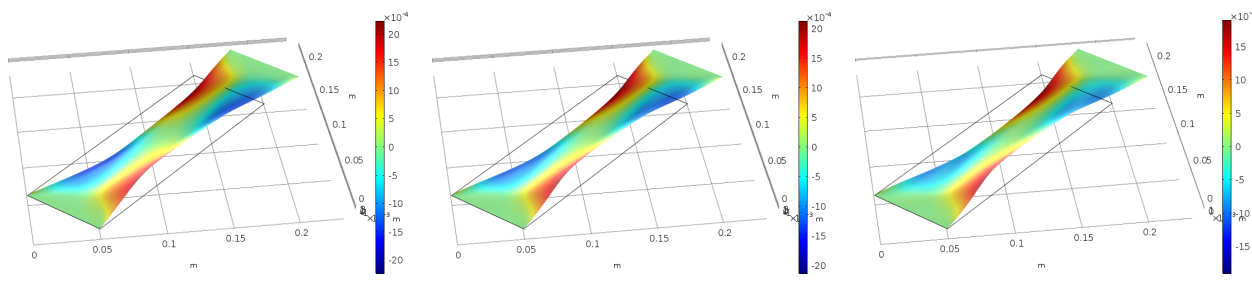

Figure 8. Reduced 2D model with pivot heights $1 \mathrm{~mm}, 2.5 \mathrm{~mm}$, and $5 \mathrm{~mm}$. The colors show the transversal displacement.

model performs better as the height of the connections increases due to their greater induced deformation.

Figure 7 depicts a chiral effect due to the particular arrangement of the microstructure. Indeed, the transverse displacement, highlighted in color, clearly reveals an overall torsion of the entire sample in the extension test. The same behavior is obtained by using the present model, as seen in Figure 8. We remark that the same effect cannot be obtained with the previously developed model [Giorgio 

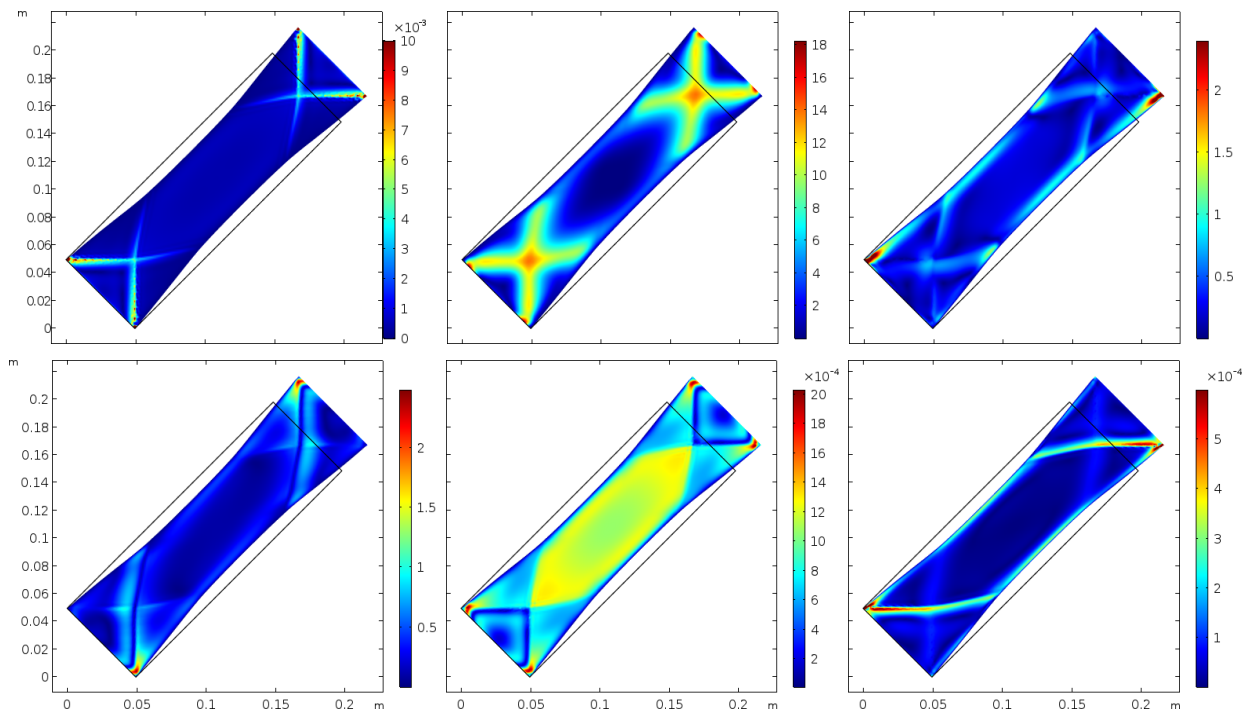

Figure 9. Reduced 2D model for height pivot $5 \mathrm{~mm}$ with an imposed displacement of $2.63 \mathrm{~cm}$ : deformation measures of fibers (from left to right and top to bottom) elongation, geodesic bending, out-of-plane bending, twisting, tangent plane shear, and out-ofplane shear.
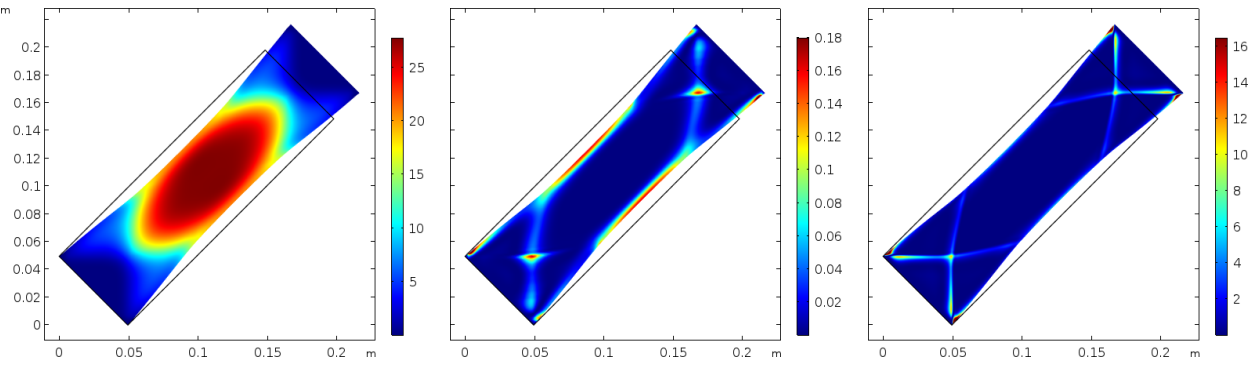

Figure 10. Reduced 2D model for height pivot $5 \mathrm{~mm}$ with an imposed displacement of $2.63 \mathrm{~cm}$ : deformation measures of pivots (from left to right) twisting, bending, and tangent plane shear. The elongation of the pivot is negligible.

et al. 2017], which does not take into account the offset of fibers and the complex deformations of the connections.

The asymmetry due to the microstructure in an extension test involving symmetric boundary conditions is also evident in the considered deformation measures, as shown in Figures 9 and 10. 

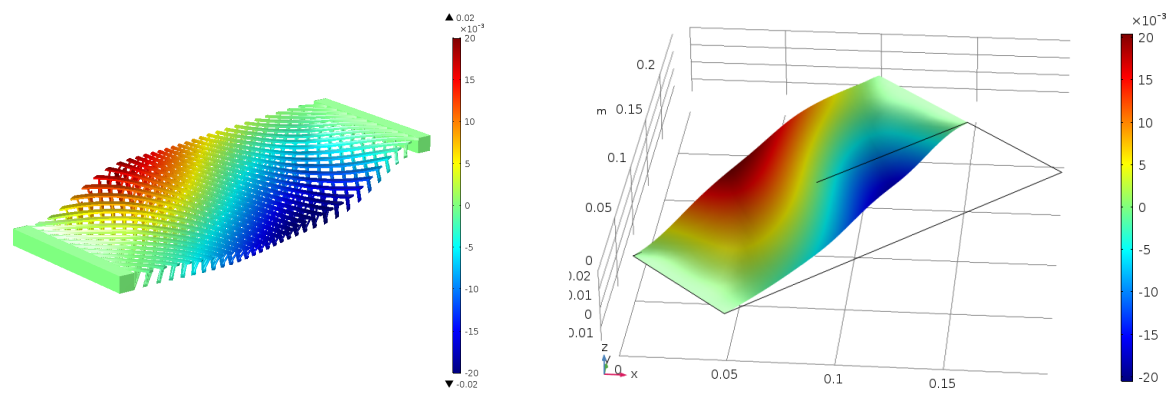

Figure 11. Buckled shapes in a shear test: Cauchy model (left) and 2D enhanced model (right).

Finally, we note that using the present 2D enhanced model, it is possible to predict the typical buckling behavior of the pantographic sheet as shown in Figure 11.

\section{Conclusions}

The comparison of the numerical results obtained by using the refined 3D model and the present reduced order model is very suggestive. In the reduced order model, it has been possible to introduce the initial and actual dimensions of the interconnecting pivots. Therefore, pivot deformation and the associated deformation energy are meaningful in both the refined and the reduced order models. Consequently a comparison of their predictive capabilities is straightforward.

The micro-macro identification which we have presented is based on the structure of the reduced order model and a fit of macroconstitutive parameters assuming the microconstitutive parameters and the predictions of the micromodel to be known.

Several research perspectives involving mathematical, numerical, and mechanical issues are opened by the present model, including the convergence of the micromodel to the macro multifield reduced order model.

We expect that the methods presented in [Pideri and Seppecher 1997; Alibert and Della Corte 2019; Abdoul-Anziz and Seppecher 2018] can be adapted to address this issue in the present context.

The issue of appropriate mechanical modeling for systems of the kind considered here presents interesting challenges that we intend to explore in future investigations. In this regard the present work may be considered a prototypical problem in the more general context of mathematical modeling of metamaterials.

Finally, it remains to investigate further optimization of the numerical strategies, the numerical codes, and the computational techniques to be used in the study of metamaterials. 
Both structural optimization and metamaterial design require the iterative solution of many deformation problems. To be able to use very efficient numerical integration schemes, specially tailored for strongly nonlinear and potentially unstable systems, is of great importance. Relevant developments are presented in [Cazzani et al. 2016; Turco et al. 2016a; 2019; 2018; Greco et al. 2019; Khakalo and Niiranen 2017; Maurin et al. 2019].

\section{Acknowledgement}

This research has been funded by the Italian Ministero dell'Istruzione dell'Università e della Ricerca (MIUR) through the PRIN 2015.

\section{References}

[Abdoul-Anziz and Seppecher 2018] H. Abdoul-Anziz and P. Seppecher, "Strain gradient and generalized continua obtained by homogenizing frame lattices", Math. Mech. Complex Syst. 6:3 (2018), 213-250.

[Alibert and Della Corte 2019] J.-J. Alibert and A. Della Corte, "Homogenization of nonlinear inextensible pantographic structures by $\Gamma$-convergence", Math. Mech. Complex Syst. 7:1 (2019), 1-24.

[Alibert et al. 2003] J.-J. Alibert, P. Seppecher, and F. Dell' Isola, “Truss modular beams with deformation energy depending on higher displacement gradients", Math. Mech. Solids 8:1 (2003), $51-73$.

[Andreaus et al. 2018] U. Andreaus, M. Spagnuolo, T. Lekszycki, and S. R. Eugster, "A Ritz approach for the static analysis of planar pantographic structures modeled with nonlinear EulerBernoulli beams", Contin. Mech. Thermodyn. 30:5 (2018), 1103-1123.

[Barchiesi and Placidi 2017] E. Barchiesi and L. Placidi, "A review on models for the 3D statics and 2D dynamics of pantographic fabrics", pp. 239-258 in Wave dynamics and composite mechanics for microstructured materials and metamaterials, edited by M. A. Sumbatyan, Adv. Struct. Mater. 59, Springer, 2017.

[Barchiesi et al. 2019a] E. Barchiesi, G. Ganzosch, C. Liebold, L. Placidi, R. Grygoruk, and W. H. Müller, "Out-of-plane buckling of pantographic fabrics in displacement-controlled shear tests: experimental results and model validation", Contin. Mech. Thermodyn. 31:1 (2019), 33-45.

[Barchiesi et al. 2019b] E. Barchiesi, M. Spagnuolo, and L. Placidi, "Mechanical metamaterials: a state of the art", Math. Mech. Solids 24:1 (2019), 212-234.

[Cazzani et al. 2016] A. Cazzani, M. Malagù, and E. Turco, "Isogeometric analysis of plane-curved beams", Math. Mech. Solids 21:5 (2016), 562-577.

[Cazzani et al. 2018a] A. Cazzani, N. L. Rizzi, F. Stochino, and E. Turco, "Modal analysis of laminates by a mixed assumed-strain finite element model”, Math. Mech. Solids 23:1 (2018), 99-119.

[Cazzani et al. 2018b] A. Cazzani, M. Serra, F. Stochino, and E. Turco, "A refined assumed strain finite element model for statics and dynamics of laminated plates", Contin. Mech. Thermodyn. (online publication September 2018).

[De Angelo et al. 2019a] M. De Angelo, E. Barchiesi, I. Giorgio, and B. E. Abali, "Numerical identification of constitutive parameters in reduced-order bi-dimensional models for pantographic structures: application to out-of-plane buckling", Arch. Appl. Mech. 89:7 (2019), 1333-1358. 
[De Angelo et al. 2019b] M. De Angelo, M. Spagnuolo, F. D’Annibale, A. Pfaff, K. Hoschke, A. Misra, C. Dupuy, P. Peyre, J. Dirrenberger, and M. Pawlikowski, "The macroscopic behavior of pantographic sheets depends mainly on their microstructure: experimental evidence and qualitative analysis of damage in metallic specimens", Contin. Mech. Thermodyn. 31:4 (2019), 1181-1203.

[dell'Isola et al. 2015] F. dell'Isola, T. Lekszycki, M. Pawlikowski, R. Grygoruk, and L. Greco, "Designing a light fabric metamaterial being highly macroscopically tough under directional extension: first experimental evidence", Z. Angew. Math. Phys. 66:6 (2015), 3473-3498.

[dell'Isola et al. 2019] F. dell'Isola, P. Seppecher, J. J. Alibert, and et al., "Pantographic metamaterials: an example of mathematically driven design and of its technological challenges", Contin. Mech. Thermodyn. 31:4 (2019), 851-884.

[Eremeyev 2018] V. A. Eremeyev, “A nonlinear model of a mesh shell”, Mech. Solids 53:4 (2018), 464-469.

[Eremeyev 2019] V. A. Eremeyev, "Two- and three-dimensional elastic networks with rigid junctions: modeling within the theory of micropolar shells and solids", Acta Mech. 230:11 (2019), 3875-3887.

[Eremeyev and Pietraszkiewicz 2006] V. A. Eremeyev and W. Pietraszkiewicz, "Local symmetry group in the general theory of elastic shells", J. Elasticity 85:2 (2006), 125-152.

[Eremeyev and Pietraszkiewicz 2016] V. A. Eremeyev and W. Pietraszkiewicz, "Material symmetry group and constitutive equations of micropolar anisotropic elastic solids", Math. Mech. Solids 21:2 (2016), 210-221.

[Eremeyev et al. 2018] V. A. Eremeyev, F. dell'Isola, C. Boutin, and D. Steigmann, "Linear pantographic sheets: existence and uniqueness of weak solutions", J. Elasticity 132:2 (2018), 175-196.

[Eugster et al. 2019] S. R. Eugster, F. dell'Isola, and D. J. Steigmann, "Continuum theory for mechanical metamaterials with a cubic lattice substructure”, Math. Mech. Complex Syst. 7:1 (2019), $75-98$.

[Giorgio 2016] I. Giorgio, "Numerical identification procedure between a micro-Cauchy model and a macro-second gradient model for planar pantographic structures", Z. Angew. Math. Phys. 67:4 (2016), art. id. 95.

[Giorgio et al. 2017] I. Giorgio, N. L. Rizzi, and E. Turco, "Continuum modelling of pantographic sheets for out-of-plane bifurcation and vibrational analysis", Proc. A. 473:2207 (2017), art. id. 20170636.

[Greco et al. 2019] L. Greco, M. Cuomo, and L. Contrafatto, "A quadrilateral $G^{1}$-conforming finite element for the Kirchhoff plate model", Comput. Methods Appl. Mech. Engrg. 346 (2019), 913-951.

[Khakalo and Niiranen 2017] S. Khakalo and J. Niiranen, "Isogeometric analysis of higher-order gradient elasticity by user elements of a commercial finite element software", Comput.-Aided Des. 82 (2017), 154-169.

[Maurin et al. 2019] F. Maurin, F. Greco, and W. Desmet, "Isogeometric analysis for nonlinear planar pantographic lattice: discrete and continuum models", Contin. Mech. Thermodyn. 31:4 (2019), 1051-1064.

[Pideri and Seppecher 1997] C. Pideri and P. Seppecher, "A second gradient material resulting from the homogenization of an heterogeneous linear elastic medium", Contin. Mech. Thermodyn. 9:5 (1997), 241-257.

[Placidi et al. 2016] L. Placidi, E. Barchiesi, E. Turco, and N. L. Rizzi, "A review on 2D models for the description of pantographic fabrics", Z. Angew. Math. Phys. 67:5 (2016), art. id. 121.

[Seppecher et al. 2011] P. Seppecher, J.-J. Alibert, and F. dell'Isola, "Linear elastic trusses leading to continua with exotic mechanical interactions”, J. Phys. Conf. Ser. 319:1 (2011), art. id. 012018. 
[Spagnuolo et al. 2017] M. Spagnuolo, K. Barcz, A. Pfaff, F. dell'Isola, and P. Franciosi, "Qualitative pivot damage analysis in aluminum printed pantographic sheets: numerics and experiments", Mech. Res. Commun. 83 (2017), 47-52.

[Steigmann and dell'Isola 2015] D. J. Steigmann and F. dell'Isola, "Mechanical response of fabric sheets to three-dimensional bending, twisting, and stretching", Acta Mech. Sin. 31:3 (2015), 373382.

[Turco et al. 2016a] E. Turco, F. dell'Isola, A. Cazzani, and N. L. Rizzi, "Hencky-type discrete model for pantographic structures: numerical comparison with second gradient continuum models", Z. Angew. Math. Phys. 67:4 (2016), art. id. 85.

[Turco et al. 2016b] E. Turco, F. dell'Isola, N. L. Rizzi, R. Grygoruk, W. H. Müller, and C. Liebold, "Fiber rupture in sheared planar pantographic sheets: numerical and experimental evidence", Mech. Res. Commun. 76 (2016), 86-90.

[Turco et al. 2018] E. Turco, A. Misra, M. Pawlikowski, F. dell'Isola, and F. Hild, "Enhanced PiolaHencky discrete models for pantographic sheets with pivots without deformation energy: numerics and experiments", Int. J. Solids. Struct. 147 (2018), 94-109.

[Turco et al. 2019] E. Turco, A. Misra, R. Sarikaya, and T. Lekszycki, "Quantitative analysis of deformation mechanisms in pantographic substructures: experiments and modeling", Contin. Mech. Thermodyn. 31:1 (2019), 209-223.

Received 23 Jul 2019. Accepted 11 Nov 2019.

IVAN GIORGIO: ivan.giorgio@uniroma1.it

International Research Center on Mathematics and Mechanics of Complex Systems,

Università degli studi dell' Aquila, L'Aquila, Italy

NiCOLA L. RIZZI: nicolaluigi.rizzi@uniroma3.it

Department of Architecture, Università degli studi Roma Tre, Roma, Italy

UGO ANDREAUS: ugo.andreaus@uniroma1.it

Department of Structural and Geotechnical Engineering, Università degli studi di Roma "La

Sapienza", Roma, Italy

DAVID J. STEIGMAnN: dsteigmann@berkeley .edu

Department of Mechanical Engineering, University of California, Berkeley, Berkeley, CA, United States 